\title{
905 FASN PREVENTS IMMUNOGENICITY OF IRRADIATED GLIOBLASTOMA BY INHIBITING ER STRESS
}

Mara De Martino*, Camille Daviaud, Claire Vanpouille-Box. Weill Cornell Medicine, New York, NY, USA

Background Glioblastoma (GBM) is the most aggressive and incurable adult brain tumor. Radiation therapy (RT) is an essential modality for GBM treatment and is recognized to stimulate anti-tumor immunity by inducing immunogenic cell death (ICD) subsequent to endoplasmic reticulum (ER) stress. However, RT also exacerbates potent immunosuppressive mechanisms that facilitate immune evasion. Notably, increased de novo lipid synthesis by the fatty acid synthase (FASN) is emerging as a mechanism of therapy resistance and immune escape. Here, we hypothesize that RT induces FASN to promote GBM survival and evade immune recognition by inhibiting ER stress and ICD.

Methods To determine if lipid synthesis is altered in response to RT, we first assessed FASN expression by western blot (WB) and lipid accumulation by BODIPY staining in murine (CT2A and GL261) and human (U118) GBM cell lines. Next, FASN expression was blocked in CT2A cells using CRISPRCas9 or an inducible shRNA directed against Fasn to evaluate ICD and ER stress markers by ELISA, WB, and electron microscopy. Finally, CT2AshFASN cells or its non-silencing control (CT2AshNS) were orthotopically implanted and FASN knockdown was induced by feeding the mice with doxycycline. The immune contexture was determined by in situ immunofluorescence ( $n=3 /$ group). Remaining mice were followed for survival ( $\mathrm{n}=7 /$ group).

Results We found that in vitro irradiation of GBM cells induces lipid accumulation in a dose-dependent fashion; an effect that is magnified over time lasting at least 6/7 days. Consistent with these findings, FASN expression was upregulated in irradiated GBM cells. Confirming the role of FASN, RTinduced accumulation of lipids was reverted when GBM cells were incubated with a FASN inhibitor. Next, we found that FASN ablation in CT2A cells induces mitochondria disruption and was sufficient to increase the expression of the ER stress makers BIP and CHOP. Along similar lines, shFASN enhances the secretion of the ICD markers HMGB1, IFN-beta and CXCL10 in irradiated CT2A cells. In vivo, CT2AshFASN tumors presented increased infiltration of CD11c+ cells and $\mathrm{CD} 8+\mathrm{T}$ cells, consistent with prolonged mice survival (56 days vs. 28 days for CT2AshNS). Importantly, $43 \%$ of CT2AshFASN-bearing mice remained tumor-free for more than 70 days, while none of the CT2AshNS-bearing mice survived.

Conclusions Altogether, our data suggest that FASN-mediated lipid synthesis is an important mechanism to prevent ER stress, ICD, and anti-tumor immune responses in GBM. While much work remains to be done, our data propose FASN as a novel therapeutic target to overcome immunosuppression and sensitize GBM to immunotherapies.

http://dx.doi.org/10.1136/jitc-2021-SITC2021.905 